\title{
DECAMETER RADIO EMISSION OF THE SUN: RECENT OBSERVATIONS
}

\author{
V. N. Melnik*, H. O. Rucker ${ }^{\dagger}$, A. A. Konovalenko*, V. V. Dorovskyy*, \\ E. P. Abranin*, and A. Lecacheux ${ }^{\ddagger}$
}

\begin{abstract}
We present an overview of the recent results in solar observations obtained at decameter radio waves using the radio telescope UTR-2. Due to up-to-date recording facilities some newly discovered phenomena in the frequency range $10-30 \mathrm{MHz}$ are given: fast type III bursts, fine time structures in normal type III bursts, solar S-bursts, type IV bursts with zebra-like fine structures, third harmonic in type II bursts and bursts in absorption of different time scale. Newly discovered properties of well known bursts such as type III bursts, type IIIb bursts, type II bursts and drift pairs were obtained too.
\end{abstract}

\section{Introduction}

The use of the new spectrometer (continuous bandwidth of $12 \mathrm{MHz}$, high frequency and time resolution of $12 \mathrm{kHz}$ and $10 \mathrm{~ms}$ respectively) on the radio telescope UTR-2 (Ukrainian T-shaped Radio telescope - second modification) (frequency band $10-30 \mathrm{MHz}$, beamwidth $25^{\prime}$ and sensitivity $5 \mathrm{Jy}$ ) allows us to strongly extend the feasibility of the studies of the solar decameter radio emission. In 2001 the first decameter type II burst was recorded. The drift rate of decameter type II bursts range from $30 \mathrm{kHz} / \mathrm{s}$ to $70 \mathrm{kHz} / \mathrm{s}$. The usual type II bursts are found to have fine structures in the form of short (durations about of 1 s) components with drift rate $0.5-3 \mathrm{MHz} / \mathrm{s}$. Type II bursts with herringbone structures with wave-like background were observed too. For the first time fine time structures in usual type III bursts were observed. They consist of sub-bursts having drift rates that can be larger or smaller than that of the parent type III bursts. Decameter S-bursts were also observed. Observational characteristics of drift pairs at $18-30 \mathrm{MHz}$ were analyzed too.

\footnotetext{
*Institute of Radio Astronomy, Ukrainian Academy of Sciences, Chervonopraporna 4, 61002, Kharkiv, Ukraine

† Space Research Institute, Austrian Academy of Sciences, Schmiedlstrasse 6, A-8042 Graz, Austria

‡ LESIA, Observatoire de Paris, UMR CNRS 8109, 92195 Meudon, France
} 

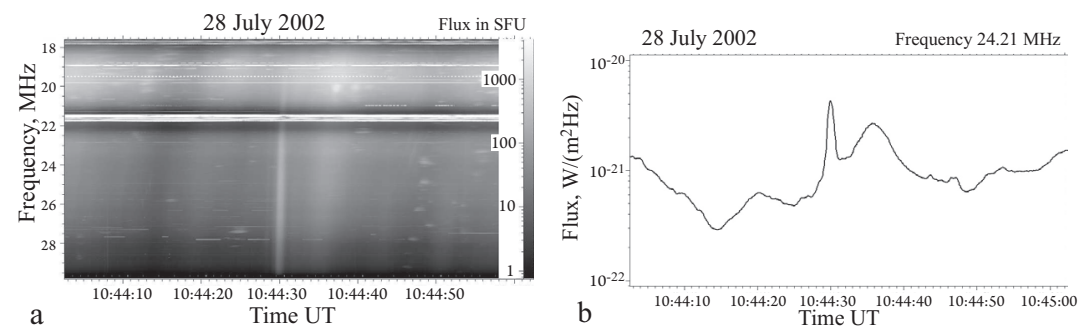

Figure 1: a) Type III-like burst (10:44:30) during type III bursts storm; b) time profiles of type III-like burst and usual type III bursts.
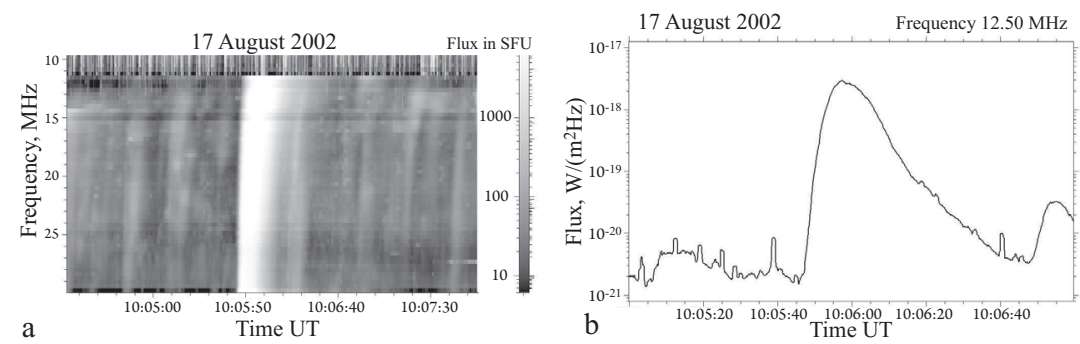

Figure 2: a) Powerful type III burst during storm of type III bursts; b) time profile of a powerful type III burst.

In this paper we briefly present some recent results (after PRE VI) obtained in the study of sporadic radio solar emissions. During this time fast type III burst (type III-like bursts), decameter type IV bursts and their fine structures in form of fiber bursts and zebrapatterns were revealed. Fine frequency structures of type IIIb bursts were considered. Decameter spikes and S-bursts as well as powerful type III bursts were analyzed too.

\section{Type III-like Bursts}

In the frequency band $10-30 \mathrm{MHz}$ we observed for the first time fast type III-like bursts [Melnik et al., 2008a] (Fig. 1a). More than 1000 such bursts were recorded in the years 2002-2006. Their drift rates $(20-30 \mathrm{MHz} / \mathrm{s})$ were sometimes higher than those of the usual type III bursts $(2-4 \mathrm{MHz} / \mathrm{s})$ in the decameter range. These bursts last $1-2 \mathrm{~s}$ and are shorter than usual type III bursts (Fig.1b). Type III-like bursts are weak bursts, in most cases their flux density is not exceeding $100 \mathrm{sfu}$. Their interesting property is related to the fact that they are observed in days when the associated active region is near the central meridian. Therefore the beaming of the radio emission of type III-like bursts is about $20^{\circ}-30^{\circ}$. Their usual high frequency drift rate can be related to the group velocity of electromagnetic waves near the emission source that is similar to the speed of 


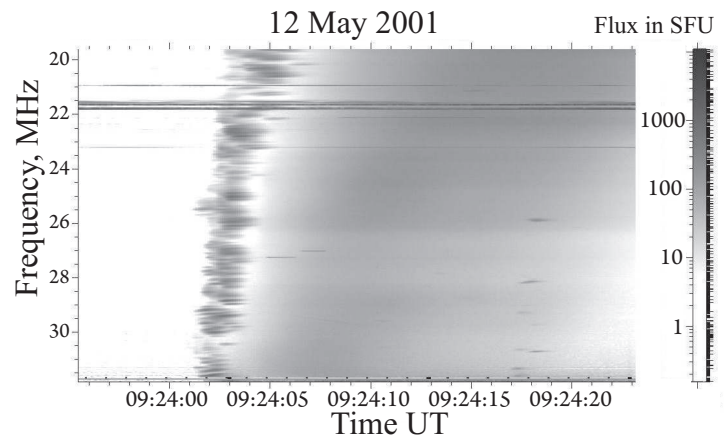

Figure 3: Type IIIb burst followed by type III burst.
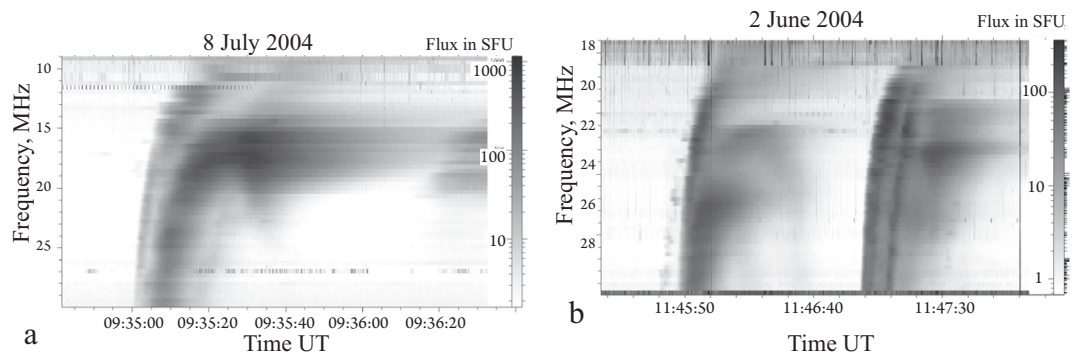

Figure 4: a) Harmonic pair of $U-$, - bursts; b) splitting $U-, \quad J$ - bursts.

fast electrons (see paper by Rutkevych and Melnik [2011a]).

\section{Powerful Type III Bursts}

We analyzed the characteristics of powerful type III bursts with flux density $>10^{-19} \mathrm{~W} / \mathrm{m}^{2} \mathrm{~Hz}$ (Fig. 2) [Melnik et al., 2011b]. The main feature of these bursts is the linear dependence of the drift rate in respect to the frequency. This means that in the radio source region of the corona the plasma density $n$ trend is exponential $(n(r) \approx \exp (-r / a)$, where $a$ is a constant). Another property of these bursts is that their duration decreases not so fast as $1 / f$, it agrees with the relationship $60 f^{-2 / 3}$.

\section{Decameter Type IIIb Bursts}

The drift rate of these bursts is similar to the one of the usual type III bursts however their duration is $3-4$ times shorter. Type IIIb bursts show fine frequency structure similar to chains of striae. The frequency bandwidth of striae is $50-70 \mathrm{kHz}$ [Melnik et 


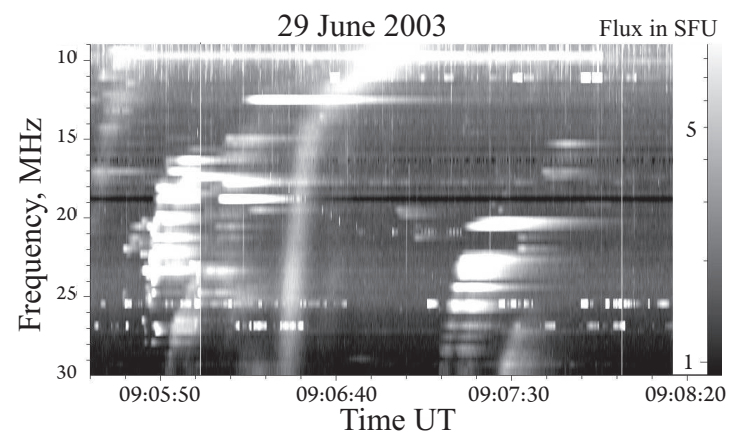

Figure 5: Dog-leg type III burst (9:06:20).

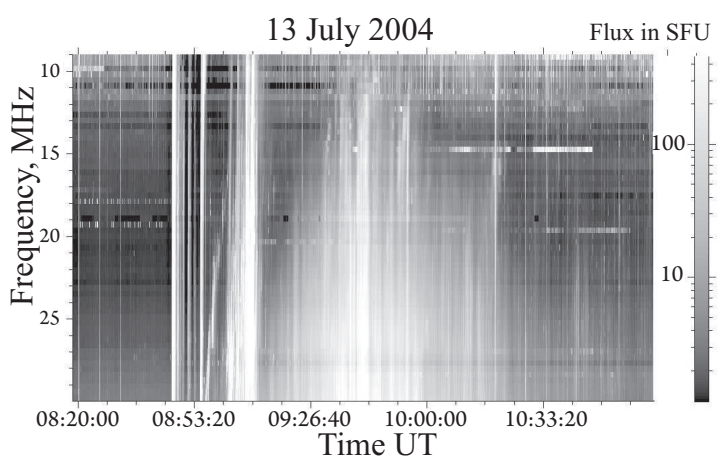

Figure 6: Decameter type IV burst.

al., 2010a], their duration and therefore the duration of type IIIb bursts is about $1.2 \mathrm{~s}$ (Fig. 3). According to some ideas these bursts can be associated with electron beams which propagate through density fluctuations in corona.

\section{Inverted Decameter U- and J-bursts}

During years 2003 - 2004 we observed in the frequency range $10-30 \mathrm{MHz}$ more than 50 inverted U- and J- bursts [Dorovskyy et al., 2010a] (Fig. 4a). That confirms the evidence of magnetic loops up to 2 - 3 solar radii in the corona. Harmonic pairs were seen not only in usual U- and J- bursts but also in Jb-bursts. There were some cases of splitting U- and J- bursts (Fig. 4b) that indicate radio emission generated in arcades of magnetic loops of different sizes. 


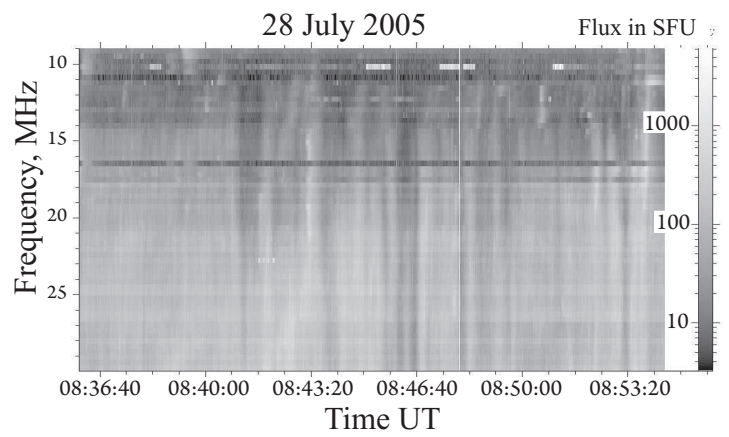

Figure 7: Fiber bursts in emission and absorption.
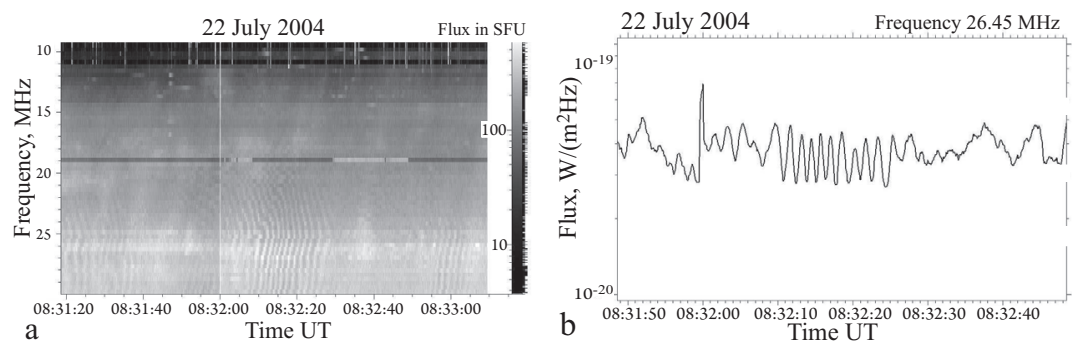

Figure 8: a) Group of zebra pattern stripes; b) equidistant stripes in zebra structure.

\section{Dog-leg Type III Bursts}

Sometimes we observed strange type III bursts which present discontinuity in drift rates and in duration at some frequency (Fig. 5) [Dorovskyy et al., 2011c]. There were more than 100 of such dog-leg type III bursts during years 2002 - 2006. The jump points are distributed in the range $13-21 \mathrm{MHz}$ with maximum at about $16 \mathrm{MHz}$. The values of the sudden change of the frequency drift rate at the jump point may reach also the factor 10. We think that the cause of such unusual type III bursts is due to coronal plasma inhomogeneity in density, temperature or both of them.

\section{Type IV Bursts}

During years 2002 - 2006 in the frequency range 10-30 MHz we recorded about 40 type IV bursts [Melnik, et al., 2008b]. The sequence of events which accompanies type IV bursts is the following: group of powerful type III bursts, phase of absorptions or large-scale burst in absorption, type II burst and finally type IV burst (Fig. 6). The type II burst can precede the type IV event or can be simultaneous with it. The duration of type IV bursts 
varies from 1 - 2 up to 6-7 hours. All the observed decameter type IV events show fine structures in form of fiber bursts in emission and absorption [Konovalenko et al., 2007; Melnik et al., 2010b] with positive and negative drift rate (Fig. 7). Fiber drift rates and their durations are close to those of usual type III bursts. In one case we recorded fine structures in form of zebra pattern (Fig. 8a) which was repeated some times during the whole type IV burst. Each such group consisted of a sequence of equidistant (up to 40) stripes (Fig 8b) [Melnik et al., 2008b]. The time interval between neighboring stripes was $1-2.5 \mathrm{~s}$ (Fig. 8b). Type II bursts associated with type IV bursts show two or sometimes even three harmonics (Fig. 9) [Melnik et al., 2008b].

\section{Decameter Spikes}

Decameter spikes are short duration bursts (life time about $1 \mathrm{~s}$ ) with bandwidth $50-70$ kHz [Melnik et al., 2011d] with flux density up to $100 \mathrm{sfu}$. Spikes are placed chaotically on the dynamic spectrum (Fig. 10a). We believe that they are generated by fast electron small density beams and that their source has a not large spatial size. The spike duration is defined by particle collision time [Melnik et al., 2011d]. This allows to estimate the temperature of the coronal plasma at frequencies $10-30 \mathrm{MHz}$. We found [Melnik et al., 2011d], using data from July 27 to August 2, 2002, that at height $1-3 R_{s}$ the plasma temperature was about $10^{6} \mathrm{~K}$. Moreover it is possible to find the angle into which fast electrons propagate along open magnetic field lines. In most cases this angle is not larger than $20^{\circ}$ [Melnik et al., 2011d].

\section{S-bursts}

S-bursts (Fig. 10b) are the shortest decameter bursts, their duration is only $0.3-0.5 \mathrm{~s}$ [Melnik et al., 2010c]. They, as type III bursts, drift from high to lower frequency, but their drift rate is 3 - 4 time smaller than those of usual type III bursts. Most S-bursts have flux density smaller than $100 \mathrm{sfu}$. Another interesting property of these bursts is the linear increase of their bandwidth with frequency. It is possible to explain the properties of these bursts and to find the magnetic field and the parameters of the background Langmuir turbulence in their source in the frame of the generation model based on coalescence of Langmuir waves with fast magnetosonic waves [Melnik et al., 2010c]. During the passage of an active region on the solar disk in July, 2002 the estimated magnetic field was $2 \mathrm{G}$.

\section{Conclusion}

Our observations of the solar radio emission in the decameter range during the period 2001-2006 gave us some important results. First of all it is to mention type IV bursts which were associated with CMEs (Coronal Mass Ejection). The obtained data fill the gap existing between meter and hectometer radio emission of the CMEs. The study of these bursts should be helpful in understanding the CME properties and the peculiarities 


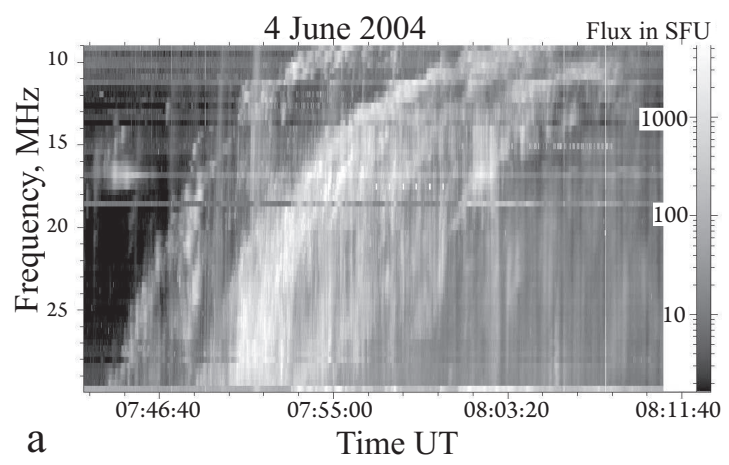

Figure 9: Type II bursts with three harmonics associated with type IV burst.
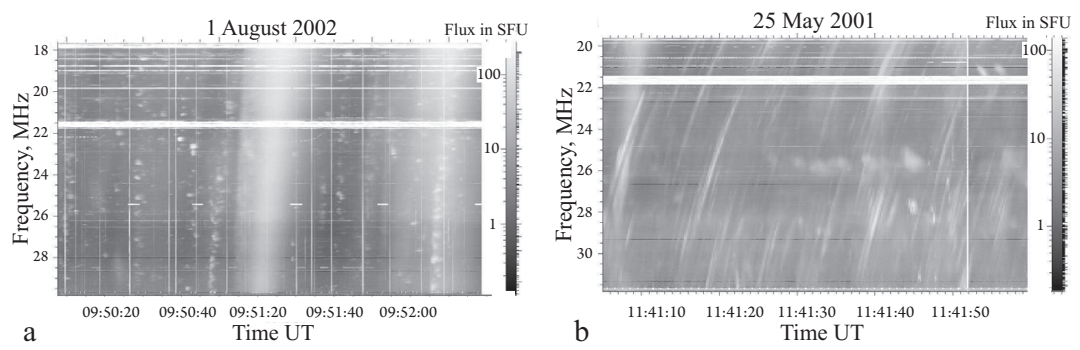

Figure 10: a) Decameter spikes chaotically located on the dynamic spectrum; b) storm of $S$ bursts.

of the interaction of CMEs with the coronal plasma. The analysis of properties of decameter spikes, type III-like bursts and S-bursts showed that they can be used as diagnostic instrument for obtaining some characteristics of the coronal plasma (density, temperature, magnetic field, background Langmuir turbulence) at height $1-3 R_{s}$ and some parameters pertinent to accelerated electrons (velocity, density, size, etc). Our observations show that the fast electron beams responsible for type III bursts can sometimes have complex nature that is manifested in type III bursts with fine time structure. Moreover we obtained that the duration of usual decameter type III bursts defines the space size of the related electrons but not the collision time. There are also some characteristics of sporadic decameter radio emissions such as fine structures in type II bursts, existence of the third harmonic in type II bursts, burst in absorption of different time scale, zebra structures in type IV, fiber bursts with positive frequency drift rates etc, which wait for interpretation and understanding. We are sure that the recent and future observations with the UTR-2 (in the frequency range $10-30 \mathrm{MHz}$ ) and the GURT (Giant Ukrainian Radio Telescope in construction near Kharkiv) (in the frequency range $10-70 \mathrm{MHz}$ ) will bring new findings in the field of solar radio emission. 


\section{References}

Dorovskyy, V.V., V.N. Melnik, A.A. Konovalenko, H. O. Rucker, E. P. Abranin, and A. Lecacheux, Solar U- and J- radio bursts at the decameter waves, ASTROPHYSICS AND COSMOLOGY AFTER GAMOW, Proc. 4th Gamow International Conf. AIP Conference Proceedings, 1206, 433-439, 2010.

Dorovskyy, V.V., V.N. Melnik, A.A. Konovalenko, H. O. Rucker, E. P. Abranin, and A. Lecacheux, Unusual Type III Bursts at the Decametre Wavelengths, in Planetary Radio Emissions VII, edited by H. O. Rucker, W.S. Kurth, P. Louarn, and G. Fischer, Austrian Academy of Sciences Press, Vienna, this issue, 359-366, 2011.

Konovalenko, A.A., A. A. Stanislavsky, E.P. Abranin, V.V. Dorovskyy, V. N. Melnik, M. L. Kaiser, A. Lecacheux, and H. O. Rucker, Absorption in Burst Emission, Solar Phys., 245, 345, 2007.

Melnik, V. N., A. A. Konovalenko, H. O. Rucker, B. P. Rutkevych, V. V. Dorovskyy, E. P. Abranin, A.I. Brazhenko, A.A. Stanislavskyy, and A. Lecacheux, Decameter Type III-like bursts, Solar Phys., 250, 133, 2008a.

Melnik, V. N., H. O. Rucker, A. A. Konovalenko, V. V. Dorovskyy, E. P. Abranin, A. I. Brazhenko, B. Thidé, A. A. Stanislavskyy, Solar Type IV Bursts at Frequencies 10-30 $\mathrm{MHz}$, in Solar Physics Research Trends, edited by P. Wang, Nova Science Publishers, New York, 287-325, 2008b.

Melnik, V. N., H. O. Rucker, A. A. Konovalenko, N. V. Shevchuk, E. P. Abranin, V. V. Dorovskyy, and A. Lecacheux, Type IIIb bursts and their fine structure in frequency band 18-30 MHz, ASTROPHYSICS AND COSMOLOGY AFTER GAMOW, Proc. 4th Gamow International Conf. AIP Conference Proceedings, 1206, 445-449, 2010a.

Melnik, V. N., A. A. Konovalenko, A. I. Brazhenko, H. O. Rucker, V. V. Dorovskyy, E. P. Abranin, A. Lecacheux, and A.S. Lonskaya, Bursts in emission and absorption as a fine structure of Type IV bursts, ASTROPHYSICS AND COSMOLOGY AFTER GAMOW, Proc. 4th Gamow International Conf. AIP Conference Proceedings, 1206, 450-454, 2010b.

Melnik, V. N., A. A. Konovalenko, H. O. Rucker, V. V. Dorovskyy, E. P. Abranin, A. Lecacheux, and A.S. Lonskaya, Solar S-bursts at Frequencies of 10-30 MHz, Solar Phys., 264, 103-117, 2010c.

Melnik, V. N., A. A. Konovalenko, H. O. Rucker, A. I. Boiko, V. V. Dorovskyy, E. P. Abranin, and A. Lecacheux, Observations of powerful type III bursts in the frequency range 10-30 MHz, Solar Phys., 269, 335, 2011a.

Melnik, V. N., N. V. Shevchuk, H. O. Rucker, A. A. Konovalenko, E. P. Abranin, V. V. Dorovskyy, A. Lecacheux, Properties of decameter spikes, Planetary Radio Emissions VII, edited by H.Ø. Rucker, W.S. Kurth, P. Louarn, and G. Fischer, Austrian Academy of Sciences Press, Vienna, this issue, 351-358, 2011b.

Rutkevych, B.P., and V.N. Melnik, Numerical Simulation of Propagation of Type III Electrons Emission, Planetary Radio Emissions VII, this issue, 391-398, 2011. 